\title{
Patient Perspectives: Valuable Food Insecurity Interventions
}

\author{
Catherine Kress, DO | Jaya Durvasula, MD | Andrea Knievel, MD, MS | Amanda M. Honsvall \\ Hoefler, MD | Lynn P. Manning, MD | Donald J. Pine, MD | Deborah Mullen, PhD | Allyson \\ Hayward, MSW
}

PRIMER. 2021;5:40.

Published: 11/3/2021 | DOI: 10.22454/PRiMER.2021.233359

\section{Abstract}

Introduction: Food insecurity (FI), defined as "limited or uncertain availability of nutritionally adequate and safe foods, or limited or uncertain ability to acquire acceptable foods in socially acceptable ways," affects over $12 \%$ of US households. Embarrassment persists for patients with $\mathrm{FI}$, and due to the potential consequences of $\mathrm{Fl}$, including increased utilization of the health care system, it is important to find causes and potential interventions for $\mathrm{Fl}$. The purpose of this project was to better understand $\mathrm{FI}$ from the patient perspective, including contributing factors, perceived health effects, and helpful interventions.

Methods: Interviews $(\mathrm{N}=21)$ were conducted with suburban community residency clinic patients who screened positive for $\mathrm{Fl}$ in the last 12 months. Six open-ended questions and a ranking question examined contributors to $\mathrm{Fl}$, effects of $\mathrm{Fl}$, perceptions of clinic intervention helpfulness, and ideas for novel interventions.

Results: Patients identified lack of income (85.7\%) as the primary issue they faced. Secondary identified issues were lack of transportation (38.1\%), too much debt (33.3\%), and food assistance programs not providing for all needs (33.3\%). Fl effects on patients' health included difficulty adhering to specialized diets and the need to modify eating patterns due to lack of food. Surprisingly, $28.6 \%$ perceived no $\mathrm{FI}$ related-health effects. Patients felt that the most valuable clinic intervention was provision of urgent need food boxes, followed by FI screening and referrals to community food resources.

Conclusions: Frequent FI screening is in itself useful to patients. Screening paired with community food resource referrals and urgent-need food boxes are the most helpful interventions according to patients.

\section{Introduction}

In $2019,10.5 \%$ of US households and $13.6 \%$ of households with children reported food insecurity (FI). ${ }^{1}$ Stigma and embarrassment are commonly associated with food insecurity, adding to the challenges of identifying and treating $\mathrm{FI}^{2}{ }^{2}$ Increased interactions with the health care system in elderly with $\mathrm{FI}$ also increases their financial burden. ${ }^{3}$ This supports the need for improvements in screening and interventions. In the last 5 years, our family medicine residency clinic identified similar rates of $\mathrm{Fl}$ in our population compared to United States Department of Agriculture data, utilizing a validated screening tool that also determined whether patients had immediate 
food needs. ${ }^{4-6}$ In a previous study, our clinic determined that patient-conducted FI screening during check-in yielded much higher positivity rates than physician-initiated screening during clinic visits, which is consistent with other studies that show patients prefer surveys or nurse-initiated screening. ${ }^{4,7}$ Broadening this understanding of $\mathrm{Fl}$ in our community, our study investigates the patient perspective on common $\mathrm{FI}$ contributing factors, health effects of $\mathrm{Fl}$, and $\mathrm{FI}$ interventions.

\section{Methods}

\section{Design}

Eligible patients from a suburban Midwestern community family medicine residency clinic were 18 years or older, English-speaking, and had screened positive for $\mathrm{Fl}$ in the last 12 months by answering yes to at least one screening question based on a previously validated two-question screening tool. 5,8 Participants were offered a $\$ 25$ grocery gift card as incentive to participate in the interview. Eligible patients $(n=100)$ were invited to participate with a goal of recruiting 30 patients. Recruitment ended early with only 21 of the planned 30 interviews, due to a lack of novel themes emerging in responses in this institutional review board-approved study during the study period of November 2018 through April 2019.

The research team of residents, attending physicians, a licensed clinical social worker, and research scientist developed seven interview questions. Questions were primarily open-ended, discretionary and conditional probes with one ranking question. The social worker trained two social work interns and a medical student in recruitment and structured interviewing. ${ }^{9}$ Interviews were conducted by an interviewer with a scribe.

\section{Analysis}

Two physician readers performed initial analysis and independently identified key words, phrases, and ideas, along with frequencies of the qualitative data. After individual coding, interrater comparisons ensured agreement in response coding. The research scientist completed a final blinded review of the coding and qualitative analysis. Item ratings of 3 or greater from the ranking question, on Likert scale items (0-not helpful to 5-extremely helpful), were classified as helpful.

\section{Results}

Of the 21 adults interviewed, $95 \%$ identified as the primary household food buyer, $33 \%$ lived in households with at least one child, and one identified as homeless. Respondents were $20-70$ years old and $71 \%$ female.

Respondents cited many contributors to FI (Figure 1). The greatest contributor was lack of money for food (85.7\%); one mentioned "medication ... is very expensive and it's difficult to budget for food." The second greatest contributor was transportation (38.1\%), with comments including "savings can let me buy in bulk, but I can't carry it on the bus." Thirty-three percent reported too much debt or that Supplemental Nutrition Assistance Program (SNAP) and women, infants, and children (WIC) food support resources were insufficient for their households' needs, one noted that they "only get \$190 dollars per year for SNAP which is not enough." Other factors affecting FI included variable income, SNAP/WIC ineligibility, frustration due to little diet variety, embarrassment about $\mathrm{FI}$, where "due to pride, family refused to accept the assistance," and unexpected costs (28.6\% each). Most respondents (71.4\%) felt that Fl affected their health, leading to modified eating patterns and making it difficult to adhere to special diets (Figure 2), whereas $28.6 \%$ did not perceive negative health ramifications from FI. Also, respondents felt that FI caused stress (23.8\%) and other negative health impacts (Figure 2).

When rating the helpfulness of current clinic interventions, $100 \%$ said emergency food boxes were helpful 
(Figure 3). One respondent said, "the food box was the best thing l've ever gotten." The FI screening process and referral to food resources were helpful or somewhat helpful (95\%) to respondents, with $28.6 \%$ reporting using food shelves after referrals. Referral to SNAP or WIC was at least somewhat helpful (75\%).

When asked for feedback about clinic interventions, respondents suggested that we should screen more often for $\mathrm{FI}$ and continue to educate patients about FI (Figure 4). Some respondents stated they were not aware clinics could help patients with FI, with one respondent stating, "I didn't know I could ask for help here." Another respondent remarked, "Maybe another staff member ... could ask right at the end before patients see the doctor." Others did not think to bring up the subject in clinic visits. They expressed appreciation for frequent screening and resources for FI (Figure 4). One respondent stated it would be helpful if the clinic would "list out the free meals that are close by." Multiple respondents suggested displaying resources in less public areas of the clinic since many people with FI are self-conscious. Another common suggestion was identification of supplemental resources, since even those qualifying for support often run out towards the end of the month.

\section{Discussion}

This study sheds light on patient perspectives of FI and clinic-based interventions. A clinic's ability to immediately address this is frequently limited. Clinics can offer referrals and food boxes to solve immediate food needs.

Results showed screening alone is valued by patients struggling with FI. Positive screening may facilitate discussions that patients might be too embarrassed to initiate. Some respondents expressed guilt about asking for help or lack of awareness about clinic resources reinforcing the need of frequent screening and additional population wide clinic Fl education/awareness campaigns.

One-third of respondents did not perceive health effects related to FI. As physicians, this disconnect between $\mathrm{FI}$ and health illustrates the importance of education regarding the effects of adequate nutrition on health. Other studies have suggested that there are poorer outcomes in both children and adults with food insecurity. This includes increased rates of nutrient deficiencies and diabetes. ${ }^{10}$ However, there are few studies regarding the financial burden of FI on patients and the health care system.

Improved FI screening provides clinics with a more realistic understanding of Fl's impact in our community and patient population. This is especially important as COVID-19 is projected to increase FI to $15.6 \% .{ }^{11}$ Given these findings, clinics could consider frequent screenings for FI with referrals to community resources and offering urgent food resources or food boxes. Other studies have proposed clinics and community resources could work together more effectively to help benefit those with FI. ${ }^{12}$

This study was limited by the small respondent group at one site with a relatively homogeneous population. Future work includes investigating correlations between health outcomes, chronic illness, financial burdens and $\mathrm{FI}$, and the role of transportation in $\mathrm{FI}$.

\section{Conclusions}

FI screening opens conversations and leads to patient use of community support. It is clear that the screening process itself is inherently useful. Screening starts conversations about $\mathrm{Fl}$ and may reduce embarrassment and/or show that clinics can be a resource. It is also important to implement interventions that reduce $\mathrm{FI}$ for our patients, such as offering food boxes for patients in immediate need.

\section{Tables and Figures}


Figure 1: Contributors to Food Insecurity

\begin{tabular}{|c|c|c|}
\hline Insufficient funds & 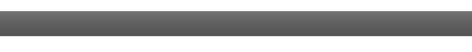 & $85.7 \%$ \\
\hline SNAP/WIC not enough & $33.3 \%$ & \\
\hline Variable income & $28.6 \%$ & \\
\hline Food shelf insufficient for needs & $28.6 \%$ & \\
\hline Embarassment/guilt asking for help & $28.6 \%$ & \\
\hline Unexpected household costs & $28.6 \%$ & \\
\hline Disability & $23.8 \%$ & \\
\hline Not aware of resources & $14.3 \%$ & \\
\hline
\end{tabular}

\section{Figure 2: Perceived Effects of Food Insecurity on Health}

\begin{tabular}{|c|c|c|c|}
\hline No perceived effect on health & & & $28.6 \%$ \\
\hline Modified eating patterns due to lack of food & & & $28.6 \%$ \\
\hline Stress & & $23.8 \%$ & \\
\hline Iron deficiency anemia & & $9.5 \%$ & \\
\hline Skipped medications & & $9.5 \%$ & \\
\hline Negative impact (unexplained) & & $9.5 \%$ & \\
\hline Weight fluctuation & $4.8 \%$ & & \\
\hline
\end{tabular}

Figure 3: Helpfulness of Current Clinical Food Insecurity Interventions ( $\mathbf{n}=21)$

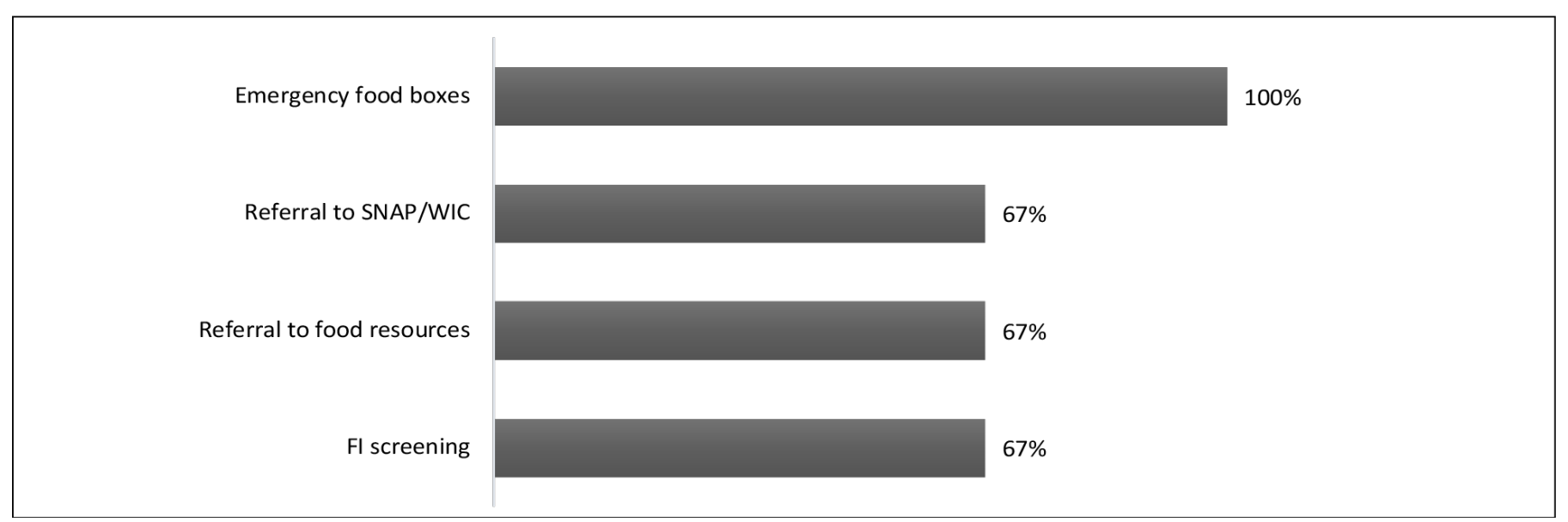


Figure 4: :Suggested Valuable Interventions Addressing FI

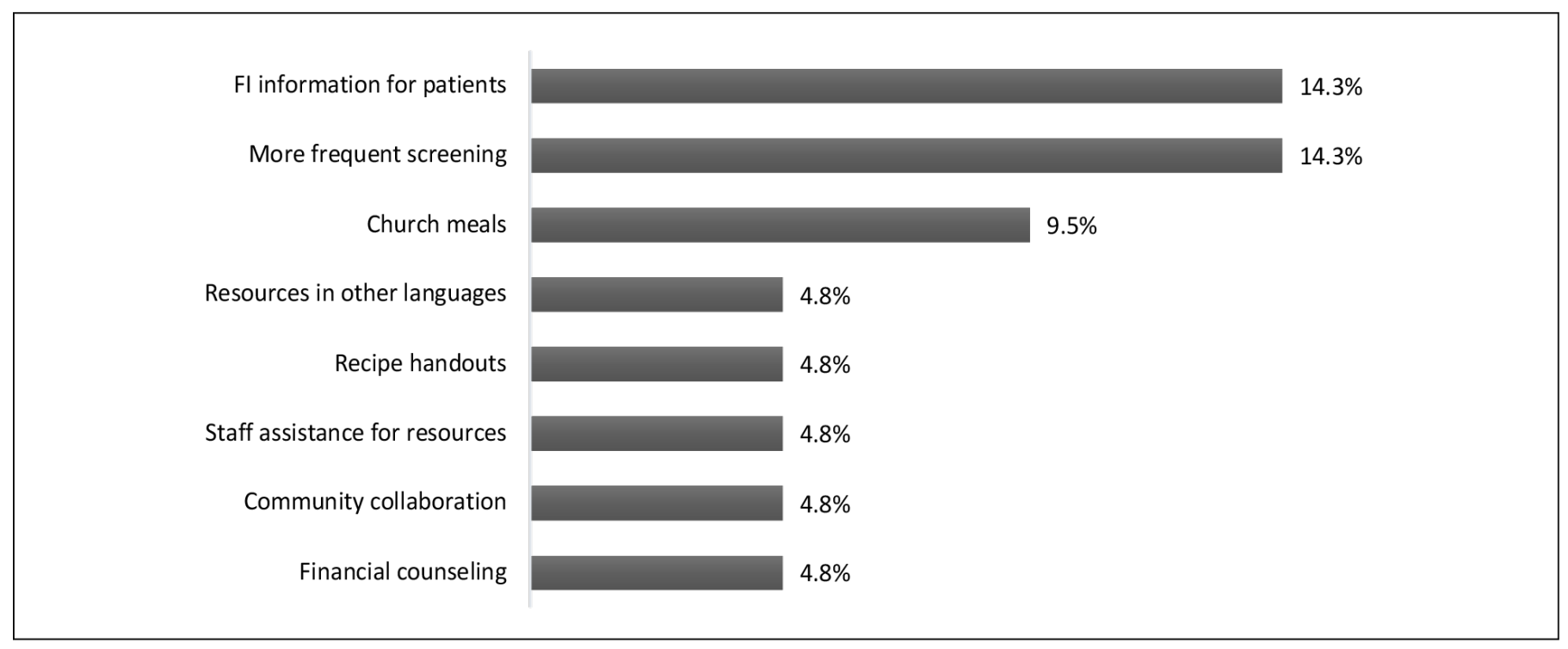

\section{Acknowledgments}

The authors thank Isaac Zoller, MD; Richard Mitchell, MD; Karvee Kawalawu, MSW, Myra Shevchenko, MSW, Lanre Adekola, MD and Beth Pearson, RN, for their assistance in the development, coordination, and execution of our research.

Financial Support: The Park Nicollet Foundation provided funding to conduct this study. Funds were utilized to cover the costs of a scribe and interviewer, including CITI training, as well as participant recruitment, data analysis, printing, and $\$ 25$ gift card incentives for respondents.

Poster Presentations: This study was presented at the following venues:

- MAFP Research \& Innovation Forum, March 2020, Bloomington, MN

- AAFP National Conference, Family Medicine residents and medical students, e-Poster, July 2020,

- STFM Annual Conference, e-Poster August 2020,

- AAFP FMX Conference, e-Poster, October 2020,

- STFM Conference on Practice Improvement, e-Poster, September 2020

- NAPCRG, e-Poster, November 2020

- FMM - e-Poster, November 2020

\section{Corresponding Author}

Catherine Kress, DO

HealthPartners Western Wisconsin Rural Family Medicine Residency, 535 Hospital Rd, New Richmond, WI 54017

catherine.a.kress@healthpartners.com

\section{Author Affiliations}

Catherine Kress, DO - HealthPartners Western Wisconsin Rural Family Medicine Residency, Amery and New Richmond, WI

Jaya Durvasula, MD - HealthPartners Western Wisconsin Rural Family Medicine Residency, Amery and New Richmond, WI

Andrea Knievel, MD, MS - HealthPartners Western Wisconsin Rural Family Medicine Residency, Amery and New 
Richmond, WI

Amanda M. Honsvall Hoefler, MD - Department of Family Medicine, Division of Sports Medicine, University of California Los Angeles, Los Angeles, CA

Lynn P. Manning, MD - University of Minnesota Methodist Hospital Family Medicine Residency Program, St Louis Park, MN

Donald J. Pine, MD - Department of Family Medicine and Community Health, University of Minnesota, Methodist Hospital Family Medicine Residency Program, St. Louis Park, MN

Deborah Mullen, PhD - Gary W. Rollins College of Business, Department of Management, University of Tennessee at Chattanooga, Chattanooga, TN

Allyson Hayward, MSW - Department of Family Medicine and Community Health, University of Minnesota, Methodist Hospital Family Medicine Residency Program, St. Louis Park, MN

\section{References}

1. Food Security and Nutrition Assistance, United States Department of Agriculture Economic Research Service Accessed January 15, 2021. www.ers.usda.gov/data-products/ag-and-food-statistics-chartingthe-essentials/food-security-and-nutrition-assistance/

2. Marpadga S, Fernandez A, Leung J, Tang A, Seligman H, Murphy EJ. Challenges and Successes with food resource referrals for food-insecure patients with diabetes. Perm J. 2019;23:18-097. doi:10.7812/TPP /18-097

3. Spitzer AK, Shenk MPR, Mabli JG. Food insecurity is directly associated with the use of health services for adverse health events among older adults. J Nutr. 2020;150(12):3152-3160. doi:10.1093/jn/nxaa286

4. Acquah 00, Honsvall Hoefler AM, Zoller I, Manning LP, Pine DJ, Mitchell RF. Improving identification of food-insecure patients in an outpatient clinic setting. PRiMER Peer-Rev Rep Med Educ Res. 2020;4:3. doi:10.22454/PRiMER.2020.115304

5. Hager ER, Quigg AM, Black MM, et al. Development and validity of a 2-item screen to identify families at risk for food insecurity. Pediatrics. 2010;126(1):e26-e32. doi:10.1542/peds.2009-3146

6. Gundersen C, Engelhard EE, Crumbaugh AS, Seligman HK. Brief assessment of food insecurity accurately identifies high-risk US adults. Public Health Nutr. 2017;20(8):1367-1371. doi:10.1017/S1368980017000180

7. Kopparapu A, Sketas G, Swindle T. Food insecurity in primary care: patient perception and preferences. Fam Med. 2020;52(3):202-205. doi:10.22454/FamMed.2020.964431

8. Kress C, Durvasula J, Knievel A, et al. Food insecurity questionnaire. STFM Resource Library; 2021. Accessed October 18, 2021. https://resourcelibrary.stfm.org/viewdocument/food-insecurityquestionnaire-1?CommunityKey=2751b51d-483f-45e2-81 de-4faced0a290a\&tab=librarydocuments

9. Kress C, Durvasula J, Knievel A, et al. Qualitative Study Questions on Food Insecurity. STFM Resource Library; 2021. Accessed October 18, 2021. https://resourcelibrary.stfm.org/viewdocument/qualitativestudy-questions-on-food?CommunityKey=2751b51d-483f-45e2-81de-4faced0a290a\& tab=librarydocuments

10. Gundersen C, Ziliak JP. Food insecurity and health outcomes. Health Aff (Millwood). 2015;34(11):1830-1839. doi:10.1377/hlthaff.2015.0645

11. The Impact of the Coronavirus on Food Insecurity in 2020. Feeding America. Updated October 2020. Accessed January 15, 2021. www.feedingamerica.org/sites/default/files/2020-10 /Brief_Local\%20Impact_10.2020_0.pdf

12. Runkle NK, Nelson DA. The silence of food insecurity: disconnections between primary care and community organizations. J Patient Cent Res Rev. 2021;8(1):31-38. doi:10.17294/2330-0698.1765 
Copyright $(\subset 2021$ by the Society of Teachers of Family Medicine 